Part of Journal of Research of the National Bureau of Standards, Volume 14, May 1935

\title{
A METHOD FOR FINDING THE ROOTS OF THE EQUATION $f(x)=0$ WHERE $f$ IS ANALYTIC
}

\author{
By Charles L. Critchfield and John Beek, Jr.
}

\section{ABSTRACT}

An expression is derived giving the roots of equations involving only functions which may be expressed in power series. The expression takes the form $x_{n}=x_{o}-$ $\frac{U_{n-1}}{U_{n}}$, where $\sum_{i=0}^{n}(-1)^{i} a_{i} U_{n-i}=0$ and $a_{r} \equiv \frac{f^{(r)}\left(x_{o}\right)}{r ! f\left(x_{o}\right)}$, and $x_{n}$ approaches the value of the root as $n$ increases without bound. Methods are given for the treatment of certain special forms. An example is given of the application of the method to the calculation of real roots.

\section{CONTENTS}

I. Introduction

II. Derivation of equations

III. Treatment of special forms

IV. Application of results

V. Summary

\section{INTRODUCTION}

The use of recursion formulas in evaluating roots of equations was described by Euler. ${ }^{1}$ Since that time the formulas and the related determinants have been presented by various authors, ${ }^{2}$ the most recent example being the series involving determinants developed by E. T. Whittaker. ${ }^{3}$ However, three of the quantities necessary to the evaluation of the $r^{\text {th }}$ term of this series are sufficient to determine the sum of the first $r$ terms, so that the series form is not the most convenient one for calculation. In the present paper the recursion formulas are developed in an elementary way, and put in a form which is convenient for calculation.

${ }_{1}$ Introductio in Analysii Infinitorum, 1, chap. 17 (1748), or the translation by J. B. Labey, p. 257 (Paris, 1796).

2 F. Cajori, A History of Mathematics, p. 227. (McMillan, London, 1919.)

3 Proc. Edinburgh Math. Soc. 36, 103 (1918), or Whittaker and Robinson, Calculus of Observations, p. 120. (Blackie and Son, London, 1924.) 


\section{DERIVATION OF EQUATIONS}

Required the value of $x$, which is a root of the equation $f(x)=0$, where $f(x)$ is analytic in the neighborhood of $x$. Then if an arbitrary approximation to $x, x_{0}$, lies in the neighborhood of $x$ the function $f(x)$ is given by the series

$$
f(x)=a_{0}+a_{1}(\Delta x)+\frac{a_{2}}{2 !}(\Delta x)^{2}+\ldots \ldots+\frac{a_{r}}{r !}(\Delta x)^{r}+\ldots \ldots
$$

where the coefficients $a_{r} \equiv f^{(r)}\left(x_{0}\right)$ are given and $\Delta x \equiv x-x_{0}$.

Define

$$
g_{n}(x) \equiv 1+b_{n, 1}(\Delta x)+\frac{b_{n, 2}}{2 !}(\Delta x)^{2}+\ldots \ldots+\frac{b_{n, n-1}}{(n-1) !}(\Delta x)^{n-1}
$$

where the $n-1$ constants $b_{n .1} b_{n, 2}-b_{n, n-1}$ are so chosen that $n-1$ consecutive derivatives (beginning with the second) of the function $\varphi_{n}(x)$ vanish at $x_{0}$, where

Then

$$
\begin{gathered}
\varphi_{n}(x) \equiv f(x) g_{n}(x)=\varphi_{n}\left(x_{0}\right)+(\Delta x) \varphi_{n}{ }^{\prime}\left(x_{0}\right)+\frac{(\Delta x)^{n+1}}{(n+1) !} \varphi_{n}^{(n+1)}\left(x_{0}\right)+ \\
\frac{(\Delta x)^{n+2}}{(n+2) !} \varphi_{n}{ }^{(n+2)}\left(x_{0}\right)+\cdots
\end{gathered}
$$

$$
\begin{aligned}
& \varphi_{n}^{\prime}\left(x_{0}\right)=a_{1}+a_{0} b_{n, 1} \\
& \varphi_{n}^{\prime \prime}\left(x_{0}\right)=0=a_{2}+2 a_{1} b_{n, 1}+a_{0} b_{n, 2} \\
& \varphi_{n}^{\prime \prime \prime}\left(x_{0}\right)=0=a_{3}+3 a_{2} b_{n, 1}+3 a_{1} b_{n, 2}+a_{0} b_{n, 3} \\
& \varphi_{n}^{(r)}\left(x_{0}\right)=0=a_{r}+\left(\begin{array}{l}
r \\
1
\end{array}\right) a_{r-1} b_{n, 1}+\cdots \cdots+\left(\begin{array}{c}
r \\
r-1
\end{array}\right) a_{1} b_{n, r-1}+a_{o} b_{n, r} \\
& \varphi_{n}^{(n-1)}\left(x_{0}\right)=0=a_{n-1}+\left(\begin{array}{c}
n-1 \\
1
\end{array}\right) a_{n-2} b_{n, 1}+\cdots+\left(\begin{array}{c}
n-1 \\
n-2
\end{array}\right) a_{1} b_{n, n-2}+a_{0} b_{n, n-1} \\
& \varphi_{n}^{(n)}\left(x_{0}\right)=0=a_{n}+\left(\begin{array}{l}
n \\
1
\end{array}\right) a_{n-1} b_{n, 1}+\cdots+\left(\begin{array}{c}
n \\
n-2
\end{array}\right) a_{2} b_{n, n-2}+\left(\begin{array}{c}
n \\
n-1
\end{array}\right) a_{1} b_{n, n-1}
\end{aligned}
$$

The symbol $\left(\begin{array}{l}r \\ i\end{array}\right)$ is used to represent the number of combinations of $r$ things taken $i$ at a time.

The equations 4 may be solved for $\varphi_{n}^{\prime}\left(x_{0}\right)$, with the result

$$
\varphi_{n}^{\prime}\left(x_{0}\right)=\frac{D_{n}}{A_{n}}
$$


where $A_{n}$ is the cofactor of $a_{1}$ in the determinant of the $n^{\text {th }}$ order having for the element of the $r^{\text {th }}$ row and $c^{\text {th }}$ column $\left(\begin{array}{c}r \\ c-1\end{array}\right) a_{r-c+1}$. The determinant may be written

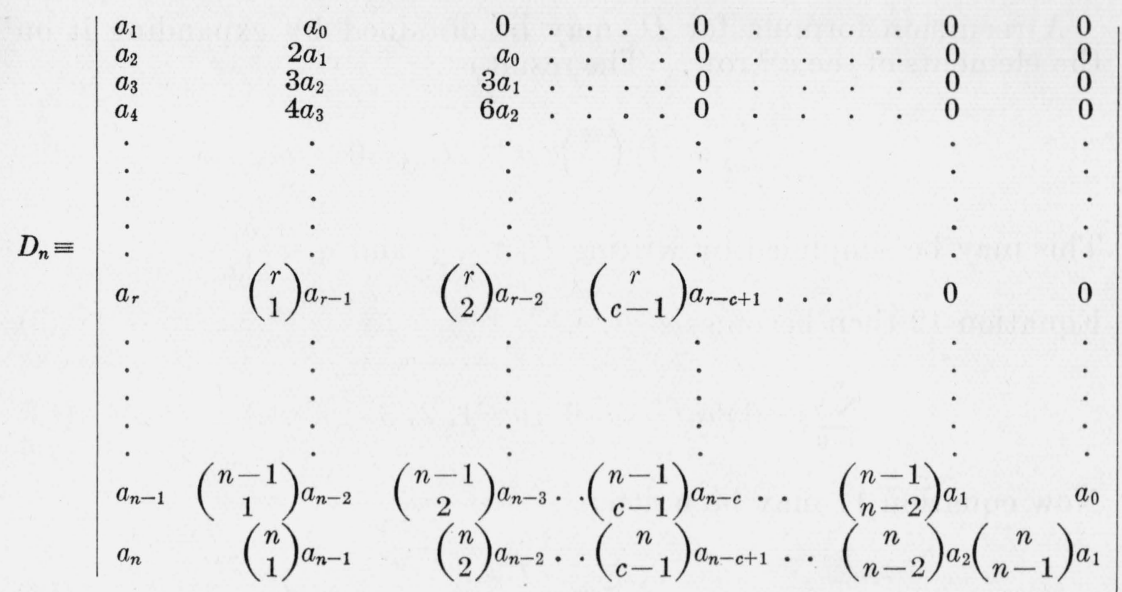

Equation 3 may be put in the form

$$
\varphi_{n}(x)=a_{0}+(\Delta x) \varphi_{n}{ }^{\prime}\left(x_{0}\right)+R_{n},
$$

where $R_{n}$ represents the sum of the terms containing powers of $\Delta x$ higher than the $n^{\text {th }}$. Solving equation 6 for $\Delta x$ and putting $\varphi_{n}(x)=0$, we have

$$
\Delta x=-\frac{a_{0}+R_{n}}{\varphi_{n}^{\prime}\left(x_{0}\right)}
$$

We may write

$$
\Delta_{n} x \equiv-\frac{a_{0}}{\varphi_{n}^{\prime}\left(x_{0}\right)}
$$

Then, as $\lim R_{n}=0, \lim \Delta_{n} x=\Delta x$.

$$
n \rightarrow \infty \quad n \rightarrow \infty
$$

From equations 8 and 5 we have

$$
\Delta_{n} x=-\frac{a_{0} A_{n}}{D_{n}}
$$

It may be shown that $A_{n}=n D_{n-1}$. Multiplying each element of $D_{n-1}$ by $\frac{r+1}{c}$, the element becomes $\left(\begin{array}{c}r+1 \\ c\end{array}\right) a_{r-c+1}$, which is the element of $A_{n}$. In this process $D_{n-1}$ is multiplied by $\frac{n !}{(n-1) !}$, or $n$. Thus

$$
A_{n}=n D_{n-1}
$$


Then from equations 9 and 10

$$
\Delta_{n} x=-\frac{n a_{0} D_{n-1}}{D_{n}}
$$

A recursion formula for $D_{n}$ may be obtained by expanding it on the elements of the $n^{\text {th }}$ row. The result is

$$
\sum_{i=0}^{n}(-1)^{i}\left(\begin{array}{c}
n \\
i
\end{array}\right) a_{i} a_{0}{ }^{i-1} D_{n-i}=0
$$

This may be simplified by writing $U_{r} \equiv \frac{D_{r}}{r ! a_{0}^{r}}$ and $\alpha_{r} \equiv \frac{a_{r}}{r ! a_{0}}$.

Equation 12 then becomes

$$
\sum_{i=0}^{n}(-1)^{i} \alpha_{i} U_{n-i}=0 \quad(n=1,2,3, \ldots)
$$

Now equation 11 may be written

$$
\Delta_{n} x=-\frac{U_{n-1}}{U_{n}}
$$

Clearly $U_{0}=D_{0}=1$, in this development, but in general the value of $U_{0}$ may be chosen arbitrarily and another value may be more convenient.

The product of $k$ consecutive convergents to $\Delta x$ is

$$
\underset{r=n+1}{r=n+k} \Delta_{r} x=(-1)^{k} \frac{U_{n}}{U_{n+k}}
$$

The limit of this product as $n$ increases without bound is $(\Delta x)^{k}$.

\section{TREATMENT OF SPECIAL FORMS}

In case $f(x) \equiv F\left(x^{2}\right)$, and $x_{0}$ is taken equal to zero, the derivatives of odd order are zero, and the functions $U_{2 m+1}$ are also zero. Thus, $\Delta_{2 m} x=0$ and $\left|\Delta_{2 m+1} x\right|=\infty$. Taking $n$ even and $k=2$ in equation 15 gives

$$
\left(\Delta_{2 m+1} x\right)\left(\Delta_{2 m+2} x\right)=\frac{U_{2 m}}{U_{2 m+2}}
$$

This expression may be evaluated, giving a convergent to $(\Delta x)^{2}$. For instance, let $f(x) \equiv \cos x=0$, and take $x_{0}=0$. Then $U_{2 m}$ becomes $\frac{E_{m}}{(2 m) !}$, where $E_{m}$ is Euler's number of order $m$, or Bernoulli's number of order $2 m$, and

$$
\left(\Delta_{2 m+1} x\right)\left(\Delta_{2 m+2} x\right)=\frac{(2 m+1)(2 m+2) E_{m}}{E_{m+1}}
$$


Now $\lim _{m \rightarrow \infty}\left(\Delta_{2 m+1} x\right)\left(\Delta_{2 m+2} x\right)=(\Delta x)^{2}$ and $\lim _{m \rightarrow \infty} \frac{(2 m+1)(2 m+2) E_{m}}{E_{m+1}}=\frac{\pi^{2}}{4}$, so that $(\Delta x)^{2}=\frac{\pi^{2}}{4}$ or $\Delta x= \pm \frac{\pi}{2}$, which gives the roots $\pm \frac{\pi}{2}$ for $\cos x=0$.

The case in which $f(x) \equiv F\left[(x-c)^{p}\right]$ and $x_{0}=c$ may be treated in a different way. Put $(x-c)^{p} \equiv z$, and find $\Delta z$ for $z_{0}=0$. Then $\Delta x=$ $(\Delta z)^{1 / p}$.

\section{APPLICATION OF RESULTS}

The familiar equation $x^{3}-2 x-5=0$ does not afford a good test of the method of this paper. If $x_{0}$ is taken as 2 , the values of $\alpha_{1}, \alpha_{2}$, and $\alpha_{3}$ are $-10,-6$, and -1 . Thus, the successive values of $U_{n}$ are integers, and may be written down immediately as far as $U_{4}$, which is sufficient for the calculation of the root correct to six decimal places.

The advantages of the method are better illustrated by the solution of a transcendental equation, such as

$$
f(x) \equiv x+\sin x+\log x+e^{x}-5=0
$$

Taking $x_{0}=1.1$, we find

$$
\begin{aligned}
& a_{0}=0.0906836 \\
& a_{1}=5.366853 \\
& a_{2}=1.28651 \\
& a_{3}=4.053 \\
& a_{4}=-0.2
\end{aligned}
$$$$
\begin{aligned}
& a_{2} / 2=0.64326 \\
& a_{3} / 6=0.6755 \\
& a_{4} / 24=-0.01
\end{aligned}
$$

TABLE 1.-Table of the products necessary for the calculation of $\Delta_{4} x$.

\begin{tabular}{|c|c|c|c|c|c|}
\hline & $\alpha_{0}=1$ & $\alpha_{1}=\frac{a_{1}}{a_{0}}$ & $\boldsymbol{\alpha}_{2}=\frac{a_{2}}{2 \alpha_{0}}$ & $\alpha_{3}=\frac{a_{3}}{6 a_{0}}$ & $\alpha_{4}=\frac{a_{4}}{24 a_{0}}$ \\
\hline$U_{0}$ & 1 & 59.18218 & 7.0934 & 7.449 & -0.1 \\
$U_{1}$ & 59.18218 & $3,502.530$ & 419.80 & 440.8 & \\
$U_{2}$ & $3,495.437$ & $206,867.6$ & 24,794 & & \\
$U_{3}$ & $206,455.2$ & $12,218,460$ & & & \\
$U_{4}$ & $12,194,116$ & & & \\
\hline
\end{tabular}

$U_{0}=1$

$U_{1}=\alpha_{1}$

$U_{2}=\alpha_{1} U_{1}-\alpha_{2}$

$U_{3}=\alpha_{1} U_{2}-\alpha_{2} U_{1}+\alpha_{3}$

$U_{4}=\alpha_{1} U_{3}-\alpha_{2} U_{2}+\alpha_{3} U_{1}-\alpha_{4}$

$$
\begin{aligned}
& \Delta_{1} x=-1 / U_{1}=-0.01690 \\
& \Delta_{2} x=-U_{1} / U_{2}=-0.0169313 \\
& \Delta_{3} x=-U_{2} / U_{3}=-0.0169307 \\
& \Delta_{4} x=-U_{3} / U_{4}=-0.0169307
\end{aligned}
$$

It may be seen that the value of $\alpha_{4}$ was not significant in the calculation of $U_{4}$. The accuracy of the calculation is not increased by the last step, as $\Delta_{3} x$ contains no error which is significant with respect to the value of $a_{0}$ used. Then $x_{3}=x_{4}=1.0830693$. The value of the root correct to eight decimal places is 1.08306929 .

It should be noted that in solving equation 6 for $\Delta x, \varphi_{n}^{\prime}\left(x_{0}\right)$ was used as a divisor. Thus, if $\varphi_{n}^{\prime}\left(x_{0}\right)$ is zero or infinity, as may be the 
case for certain values of $x_{0}$ and $n$, equation 7 does not hold. Generally this difficulty may be avoided by taking a higher value of $n$. $\varphi_{n}^{\prime}\left(x_{0}\right)$ will not ordinarily be zero, however, if $x_{0}$ is a good approximation to a root.

As the derivation of equations 13 and 14 does not involve any assumptions other than that $f(x)$ is analytic, the equations hold for complex values of the coefficients or the roots. In the evaluation of complex roots of real equations it is convenient to use a complex value for $x_{0}$, as equation 15 must be used if $x_{0}$ is real. This treatment is not satisfactory, as the sequence $U_{n} / U_{n+k}$ does not usually converge rapidly in such a case, and also because extraneous roots may be introduced.

\section{SUMMARY}

An expression is derived giving the roots of equations which involve only functions which may be expressed as power series. The expression involves only the values of the function and its derivatives at a given value of the variable.

Methods are given for the treatment of certain special forms.

An example is given of the application of the method to the evaluation of real roots.

Washington, November 22, 1934. 